\title{
The Rice Haptic Rocker: skin stretch haptic feedback with the Pisa/IIT SoftHand
}

\author{
Edoardo Battaglia ${ }^{1}$, Janelle P. Clark ${ }^{2}$, Matteo Bianchi ${ }^{1}$, \\ Manuel G. Catalano ${ }^{1,3}$, Antonio Bicchi ${ }^{1,3}$ and Marcia K. O'Malley ${ }^{2,4}$
}

\begin{abstract}
Myoelectric prostheses have seen increased application in clinical practice and research, due to their potential for good functionality and versatility. Yet, myoelectric prostheses still suffer from a lack of intuitive control and haptic feedback, which can frustrate users and lead to abandonment. To address this problem, we propose to convey proprioceptive information for a prosthetic hand with skin stretch using the Rice Haptic Rocker. This device was integrated with the myo-controlled version of Pisa/IIT SoftHand and a size discrimination test with 18 able bodied subjects was performed to evaluate the effectiveness of the proposed approach. Results show that the Rice Haptic Rocker can be successfully used to convey proprioceptive information. A Likert survey was also presented to the experiment participants, who evaluated the integrated setup as easy to use and effective in conveying proprioception.
\end{abstract}

\section{INTRODUCTION}

Restoring hand functionality in upper limb amputees is a very challenging task, with the high dexterity, versatility, sensitivity, and ease of use of a natural human hand being extremely difficult to reproduce in artificial hands. In the past, most artificial hands used in clinical practice were either purely cosmetic or body powered [1], with the actuation of the end effector realized typically through cables pulled by the shoulder. Body powered prostheses have the advantage of being simple and intrinsically able to partially convey haptic feedback to the user through the actuation; however they can also suffer from lack of comfort and smaller grip forces compared to healthy hands [2].

More recently, myoelectric prostheses, where the actuation obtained through motors is controlled by electro-myographic (EMG) signals generated by the user's muscles, are becoming increasingly popular. This approach has a high potential for better hand functionality, while also retaining a good cosmetic value, but is often difficult to control for the user [2], [3]. Moreover, when compared to body powered prostheses, myoelectric prostheses lack inherent haptic feedback, which is a highly desired feature amongst users [4]-[6] and has

\footnotetext{
${ }^{1}$ Edoardo Battaglia, Matteo Bianchi, Manuel G. Catalano and Antonio Bicchi are with Centro di Ricerca "E. Piaggio", University of Pisa, Pisa, Italy, 56126 e.battaglia, matteo.bianchi, manuel.catalano, bicchi@centropiaggio.unipi.it

${ }^{2}$ Janelle P. Clark and Marcia K. O'Malley are with the Department of Mechanical Engineering, Rice University, Houston, Texas, 77251 janelle.clark, omalleymerice.edu

${ }^{3}$ Manuel G. Catalano and Antonio Bicchi are with the Department of Advanced Robotics, Istituto Italiano di Tecnologia, Via Morego 30, 16163, Genova, Italy

${ }^{4}$ Marcia O'Malley is a Senior Scientist with TIRR-Memorial Hermann, Houston, Texas, 77030
}

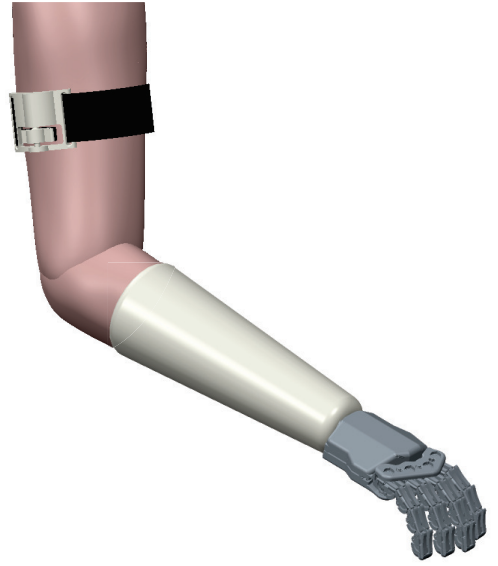

Fig. 1: Envisioned integration of the Rocker and the SoftHand.

been shown to increase embodiment of the prosthesis [7]. This critical absence can generate frustration for the user and cause abandonment of the prosthesis, which is still observed in many cases and represents a serious issue [8].

To address this deficiency, researchers have been trying to devise ways to convey haptic feedback to prosthetic users, with different methods being proposed, both invasive and non invasive [9]. Non-invasive solutions traditionally rely on sensory substitution techniques, with vibrotactile [10], electrotactile [11], force feedback [9], [12] and skin stretch [13] feedback being conveyed to the user by external devices. While different types of feedback devices are useful to convey information on different measurements, simultaneous display of different types of haptic information can also be confusing for the user [14]. For this reason it is important to focus on conveying information which is most important for task execution.

A common requirement from amputees is to be able to operate prostheses without constant visual attention [4], [15], and proprioceptive feedback has been shown to improve targeting accuracy under non-sighted conditions [16]. Because of this, in this work we choose to focus on proprioceptive feedback, and in particular on conveying it through skin stretch. In the following sections we will first describe the motivation and background behind our work, discussing some solutions that have been used in the past for proprioceptive feedback. We will then present the integration of a skin stretch haptic feedback device, the Rice Haptic Rocker, with a myo-controlled version of the Pisa/IIT SoftHand, an under- 
actuated and adaptable artificial hand which has recently been adapted for prosthetic use [17]. Finally, an experimental evaluation will be presented where we test the effectiveness of the setup at conveying proprioceptive information on hand opening with able bodied subjects in a size discrimination task. The methods used for the evaluation procedure are inspired by [18]. It is worth noting that the main focus of this work is to show that the Rice Haptic Rocker is a valuable means for proprioceptive feedback, and that the effectiveness of the device has not been ever tested before in a systematic manner. This paper aims at bridging this gap presenting the device and discussing results from a set of experiments performed with able bodied subjects.

\section{Motivation AND BACKGROUND}

From retrieving something out of a pocket to reaching to turn off an alarm clock, we encounter a multitude of tasks where we depend on our sense of proprioception. This natural mechanism is missing for myoelectric prosthetic users, who have to rely heavily on vision to know the pose of their artificial hand.

Previous work has shown that artificial introduction of proprioceptive feedback in artificial hands could be beneficial to the user. Blank et al. [16] found in a study with able-bodied subjects that proprioceptive feedback improves targeting accuracy in nonsighted and, for some tasks, also sighted conditions. More recently, in [19] an experiment was performed where participants controlled a cursor though EMG signals, with and without proprioceptive feedback, and results showed that proprioceptive feedback significantly improved myoelectric control in nonsighted conditions.

Proprioceptive feedback can be conveyed both in an invasive and non invasive fashion. Invasive approaches include Peripheral Nerve Stimulation (PNS), which uses neural electrodes to directly stimulate nerves, and can successfully deliver proprioceptive sensations [20] but is still challenging from a technological point of view [21], and its feasibility as a long term solution has yet to be proven [22]. A non-invasive option to relay proprioception is offered by sensory substitution by means of wearable haptic displays. Vibrotactile displays are the traditional approach, often evaluated in EMG control of virtual hands [23], and due to their small size and low cost of the actuators this type of feedback is often used as a baseline to evaluate other solutions [21]. However, not all of the studies conducted on vibrotactile as a feedback method for proprioception have shown promising results [24], and other solutions such as electrotactile feedback [11], [25] and skin stretch have been tested [26], [27].

\section{DEVICES AND INTEGRATION}

In this study we follow the skin stretch approach by focusing on a rocker design. Chinello et al. studied combining multiple rockers interacting in a bracelet about the forearm [28] to direct able bodied subjects in more complex wrist movements, but did not consider prosthetic applications. Our group has previously explored this mode of skin stretch mapped to a gripper aperture, [29]-[31], with prototype versions of a rocker design for providing feedback. Here,

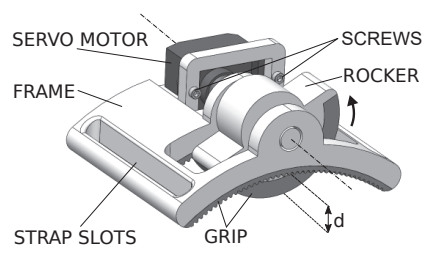

(a) CAD model and parts. The offset $\mathrm{d}$ is used to apply pressure of the user's arm.

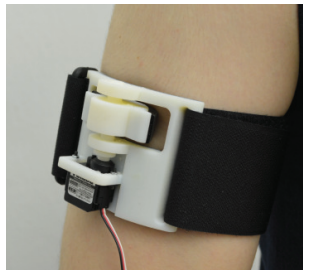

(b) Physical prototype on the upper arm of a subject.
Fig. 2: The Rice Haptic Rocker.

we propose a refined rocker solution featuring a frictional interface, which can be used without adhering the contact interface to the skin, with some advantages compared to the adhesive elements used in other studies (e.g. [27]): namely, the frictional contact interface allows easier donning when integrated with a prosthetic socket, and the rocking motion provides an intuitive cue when mapped to the hand aperture. To the best of the authors' knowledge, this is the first paper to present a skin stretch rocking device to convey information on the opening of a prosthetic hand.

The Pisa/IIT Softhand was chosen to be used as a prosthetic hand, building upon previous work on its adaptation to myoelectric control [32], [33] and use with haptic feedback devices [32], [34]-[36]. Because of its adaptability and ease of control, and because the encoder reading from the motor can be used directly to control the Rice Haptic Rocker, integration of the two devices was natural and has the potential of being profitably used in prosthetics, as we will show in the following sections.

\section{A. The Rocker}

The Rice Haptic Rocker is a wearable device that uses the sense of touch in the upper arm as a surrogate for proprioception in the prosthetic hand grasp position. The Haptic Rocker has a simple design consisting of a frame, strap, rocker, and servo, as shown in Figure 2a, with a total weight of only $60 \mathrm{~g}$. The frame, 3D printed on a Connex Objet 260, houses the rocker and servo, and is attached to the arm with a 2 inch Velcro strap. It has a curved bottom to rest comfortably on the arm, with a $3 \mathrm{D}$ printed rubber grip to keep it in place during use. The rocker has a radius of curvature of $20 \mathrm{~mm}$ with a 3/16 inch $(5 \mathrm{~mm})$ neoprene foam strip to avoid slipping and increase comfort.

The axis of rotation for the rocker is set so the contact point of the rocker has a $10 \mathrm{~mm}$ offset, $d$, from the bottom of the frame. This offset serves to create a normal force while reducing the tightness of the strap. The rocker is held in the frame by two shaft supports, and is driven by a digital servo (Futaba S3154), which is secured to the frame with 2 socket head screws and nuts (M1.6 x $0.35 \mathrm{~mm}$ ). Figure 2b shows an image of the device on a subjects upper arm. The rocker rests in the neutral position shown in Figure $2 \mathrm{~b}$ when the hand is completely open, and rotates up to 60 degrees when the hand is closed as shown in Figure $2 \mathrm{a}$, stretching the skin as the rocker rotates (for a maximum displacement of about 10.5 
$\mathrm{mm})$. This maximum value of the angle is chosen to avoid slipping on the skin and is kept constant for all subjects.

\section{B. The Pisa/IIT SoftHand}

The Pisa/IIT Softhand design [37] takes inspiration from neuroscience. It is known that humans control their hands not merely by acting on each of the numerous degrees of freedom, but rather by coordinating and co-activating them in organized motions called synergies [38], [39]. In more recent work [40], soft synergies were introduced, where the synergy serves as a reference position for a virtual hand, and the interaction forces between the hand and a grasped object depend on the stiffness matrix connecting the virtual and real hand position.

The Pisa/IIT SoftHand combines compliance and synergy inspiration into an artificial hand with 19 DoFs, 4 on each of 4 fingers, and 3 on the thumb. The fingers are capable of flexion/extension as well as ab/adduction. Traditional revolute joints were employed for ab/adduction of the fingers and at the equivalent of the carpometacarpal joint of the thumb. The rest of the joints incorporate rolling contact joints with elastic ligaments, which ensure physiologically correct motions when actuated, but easily disengage on impact to allow safe interaction with humans without compromising the hand. The elastic ligaments also allow deformation while ensuring the hand returns to its original configuration. A single tendon runs though all joints to simultaneously flex and adduct the fingers upon actuation.

The hand is actuated by a single DC motor which moves the fingers on the path of the first synergy as described in [38]. However, due its compliant design, it can conform around a large variety of objects. The motor employed in the current release is a 15 Watt Maxon DCX 22S with a GPX22 (86:1) gearhead and a 12 bit magnetic encoder, resolution of $0.0875^{\circ}$ (Austrian Microsystems). With this setup the hand has a maximum force of $130 \mathrm{~N}$ perpendicular to the palm. The CAD model of the Pisa/IIT SoftHand, as well as the design of the electronic board that is used to control it are open source and available at the Natural Machine Motion Initiative Website (http://www naturalmachinemotioninitiative.com/).

\section{Integration}

Due to the simple design of the Rocker and synergistic actuation of the SoftHand, haptic feedback can be conveyed directly by mapping the encoder reading of the SoftHand to the commanded motor position of the servo. An Arduino Uno control board is used to actuate the servo motor, and integration with the SoftHand is done in Matlab through Simulink. The servo position is controlled between 0 and 60 degrees, with the flat side of the rocker being parallel to the upper face of the servo in the zero position.

Commanded angles for the servo are obtained by linearly mapping the hand encoder reading, which assumes values inside a certain range. These values were estimated from a preliminary test observing encoder readings during a series of ten close-open cycles of the hand from the resting open position (Figure 3).

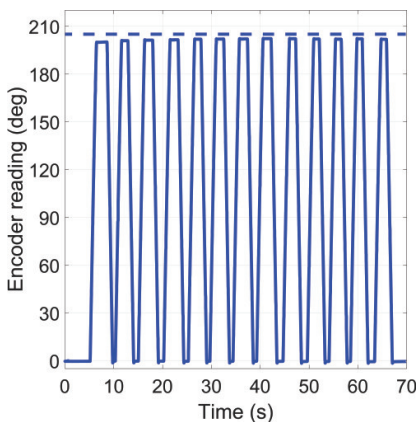

(a) Test with the SoftHand to evaluate encoder range.

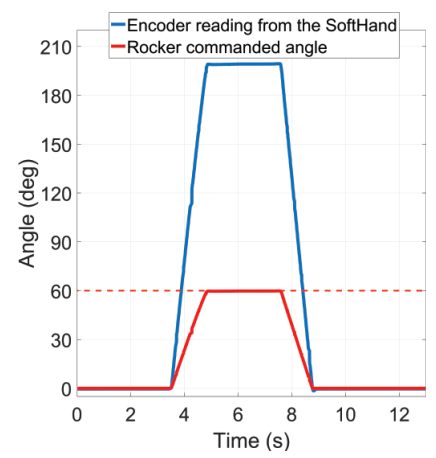

(b) Rocker angle command for a single close-open cycle.

Fig. 3: Integration of the Rice Haptic Rocker with the Pisa/IIT SoftHand. The blue dashed line in Figure $3 \mathrm{a}$ is the estimated maximum threshold for the encoder reading ( $205 \mathrm{deg}$ ), while the red dashed line in Figure $3 \mathrm{~b}$ is the maximum commanded angle for the Rocker (60 deg).

\section{EXPERIMENTAL PROTOCOL}

Eighteen healthy subjects (age $22 \pm 0.5$ years, 6 female) took part in the experiment. Two subjects were left handed, while the remaining participants were right handed. The participants did not suffer from any physical or cognitive impairment, which could interfere with their ability to follow the instructions of the study, nor any pathology that could affect tactile sensibility or muscular activity of the forearm. The methods and procedures described in this paper were carried out in accordance with the recommendations of the Institutional Review Board of Rice University with written informed consent obtained from all subjects.

The experiment was designed to evaluate the effectiveness of the proprioceptive feedback provided by the Rocker when using the SoftHand for an object size discrimination task, and was inspired by the procedure presented in [18]. Subjects were seated comfortably in front of a table. The Rocker was fastened around the right upper arm with Velcro bands for 9 subjects under the Haptic Feedback (HF) condition, while the remaining nine in the No Haptick Feedback (NHF) condition did not wear the device. The two left handed subjects were randomly assigned to each group, and female and male participants were equally distributed. The SoftHand was secured to the right hand and forearm using a handle structure with Velcro bands, and the EMG electrodes were positioned on the Flexor Digitorum Superficialis (FDS) and on the Extensor Digitorum Communis (EDC) and held in place with medical tape [9]. Before the experiment began, subjects were guided through a calibration procedure for the EMG electrodes to ensure that each person could control the opening and closure of the hand easily [33]. Participants who were under the HF condition were also instructed through some preliminary training with the Rocker, which included closing the SoftHand to a certain posture, opening it and then taking it back to the previous closure with their eyes closed.

The experiment was divided in three phases, all taking place in the same session. First was a training phase, where 


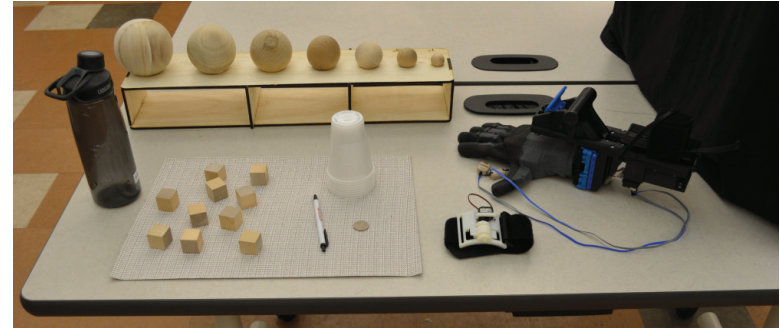

Fig. 4: From right to left: the SoftHand together with the EMG electrodes used to control it, the Rice Haptic Rocker and the objects used during the training session.

subjects learned how to use the SoftHand to interact with object of various sizes. In particular the subjects were asked to complete tasks such as building a pyramid with blocks, grasping a bottle as if they were drinking from it, grabbing a set of nested ridged cups with the SoftHand and removing them from the stack one by one using their left hand, picking up a pen and a coin from the table, and placing spheres of different sizes on a stand. An overview of the objects used in the experiment can be seen in Figure 4. Preliminary setup and training took on average about 35 minutes.

During the testing phase, which lasted on average around 20 minutes, the subjects wore noise canceling headphones, and their right arm, laying next to the SoftHand, was occluded from view by a black curtain. Pink noise was played through the headphones in order to cover possible auditory cues produced by the actuators of the SoftHand and the Rocker. Figure 5 shows the experimental setup. Subjects were presented ten different pairs of spheres and asked to tell whether the second sphere was bigger, smaller or of equal size with respect to the first sphere. Three spheres were used in this experiment with a diameter of 1.5, 2.5 and 3 inches (i.e. 38.1, 63.5 and $76.2 \mathrm{~mm}$ ), as well as a fourth grasping condition where the hand was empty. To avoid artifacts in the results due to time error, each pair of spheres was presented two times, in opposite, for a total of twenty trials in random order. During each trial, the subject had to voluntarily close the SoftHand to grasp each sphere and, depending on their group assignment, relied on the feedback from the Rocker to infer the size of the sphere.
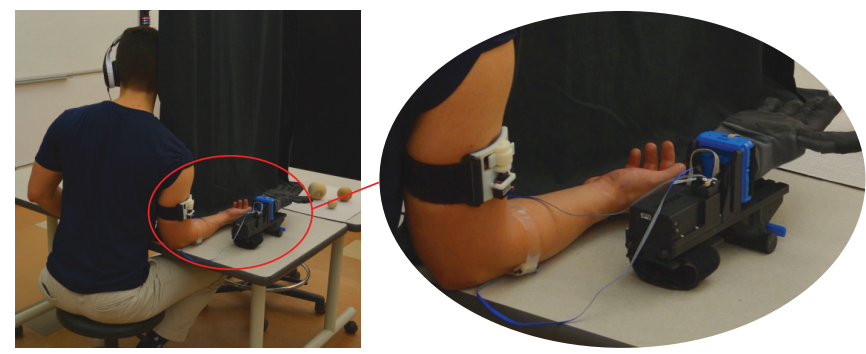

Fig. 5: Experimental setup for the object size discrimination task.

In the final evaluation phase, the participant took off the headphones and moved the SoftHand back to the other side of the curtain, where they had the possibility to use it for an additional ten minutes in a reduced version of the training procedure (limited to building a pyramid with blocks and placing spheres on the stand). Members of the HF group had the Rocker taken off for this phase, while members of the the NHF group had it placed on their upper arm. At the end of the session, the subjects took off the devices and completed a Likert-type seven point survey. The questionnaire considered the comfort and usability of the proposed experimental setup (four questions), the perceived performance (eight questions), the experimental conditions (four questions) and the level of engagement of the subjects (two questions). To each question the subject had to answer by choosing a value between 1 ("strongly disagree") and 7 ("strongly agree"), with 4 as a neutral term corresponding to "undecided". The statements were presented in pairs where one had an opposite meaning with respect to the other to check consistency and prevent bias effects from the wording.

\section{RESULTS}

The experiment required subjects to discriminate between different sphere sizes. Proprioceptive haptic feedback was conveyed by the Rocker for the HF group, while subjects under the NHF condition served as the control group. Figure 6 shows discrimination accuracy for each subject, with blue bars showing the accuracy for subjects under the HF condition and red bars showing accuracy for subjects under the NHF condition. Subject 15, despite showing an unusually high accuracy for being under the NHF condition, reported being under sound experimental conditions, which lead us to treat this result as a statistical outlier. The average accuracy for subjects under the HF condition was $73.3 \pm 11.2 \%$, well above the $33.33 \%(1 / 3)$ chance level, while for subjects under the NHF condition it was $33.3 \pm 12.7 \%$. Overall accuracy for each pair presented was also considered, with better results for the HF condition as shown in Figure 7.

Statistical analysis was performed to test for significance in the differences found. Normality of the data was tested with the Shapiro-Wilk test, from which the assumption of normality is met for Group I $(p=.82)$. However, Group II had a significant result $(\mathrm{p}=.006)$ prompting us to utilize nonparametric statistical tests for further analysis. The Kruskal-Wallis test shows a highly significant difference in discrimination accuracy $(\mathrm{p}<.001)$ between the two groups. We can thus conclude that the Haptic Rocker enables the subject to detect a difference in objects size with better accuracy than chance.

Table I shows an overview of scores for the Likert scale survey. Results were analyzed with the Kruskal-Wallis test, comparing the HF and NHF groups. According to these results, the integration of SoftHand and Rocker was deemed easy to use (Q1), able to convey information on the opening of the hand (Q9,Q10) and improve performance overall $(\mathrm{Q} 5, \mathrm{Q} 6)$. Participants under the HF condition seemed to find the object size discrimination task somewhat challenging, while subjects under the NHF condition found it understandably very difficult $(\mathrm{Q} 7-\mathrm{p}=0.051$ close to significance, Q8 - p $<0.001)$, since they were not wearing the Rocker 
I was feeling uncomfortable while using the SoftHand together with the Rocker.

$4.00(3.00 ; 4.50)$

$4.00(3.00 ; 4.50)$

The sensation provided by the Rocker on the arm felt unpleasant

I had the feeling of performing better while receiving position feedback by the Rocker.

$6.00(4.50 ; 6.50)$

$3.00(1.50 ; 3.50)$
$\mathbf{3 . 0 0}(\mathbf{3 . 0 0} ; \mathbf{5 . 0 0})$

I had the feeling of performing better when I was not receiving any feedback by the Rocker.

$3.00(3.00 ; 5.00$
$\mathbf{3 . 0 0}(3.00 ; 5.00$

Discriminating the spheres without looking at them was very difficult.

When I was using the Rocker, I was able to tell how open the SoftHand was without looking at it.

When I was using the Rocker, I had no clue about the opening of the SoftHand.

It was easy to feel the rotation of the Rocker.

It was not easy to feel the rotation of the Rocker.

During the discrimination task, I was not able to see the SoftHand, the Rocker or the spheres.

During the discrimination task, I was able to see the spheres or the devices.

During the discrimination task, I was well isolated from external noises.

During the discrimination task, I was able to hear the sounds made by the motors of the devices.

I would have been happy to continue the experiment for longer.

$5.00(5.00 ; 6.00)$

$2.00(2.00 ; 3.00)$

$6.00(5.00 ; 7.00)$

$2.00(1.00 ; 3.00)$

$7.00(7.00 ; 7.00)$

$1.00(1.00 ; 1.00)$

$7.00(6.00 ; 7.00)$

$1.00(1.00 ; 1.50)$

$6.00(4.50 ; 6.00)$

$2.00(1.50 ; 5.00)$

$2.00(1.00 ; 5.00)$

$5.00(3.50 ; 5.50)$

$5.00(4.00 ; 6.50)$

$3.00(1.00 ; 3.00) \quad 0.645$

$1.00(1.00 ; 2.50) \quad 0.051$

$\mathbf{7 . 0 0}(\mathbf{7 . 0 0} ; \mathbf{7 . 0 0}) \quad<0.001$

$5.00(4.50 ; 5.50) \quad 0.36$

$2.00(1.50 ; 2.00) \quad 0.246$

$\mathbf{5 . 0 0}(\mathbf{3 . 0 0} ; \mathbf{5 . 0 0}) \quad \mathbf{0 . 0 6 3}$

$\mathbf{3 . 0 0}(2.50 ; 5.00) \quad 0.061$

$7.00(7.00 ; 7.00) \quad 0.317$

$1.00(1.00 ; 1.00) \quad 0.317$

$\begin{array}{ll}7.00(7.00 ; 7.00) & 0.27\end{array}$

$1.00(1.00 ; 1.00) \quad 0.539$

$6.00(5.00 ; 6.50)$

$3.00(1.00 ; 5.00) \quad 0.784$

TABLE I: Results of the Likert scale survey. The central tendency of responses is summarized by using median with dispersion measured by IQR $\left(25^{\circ} \sim 75^{\circ}\right)$. Scores with significant or close to significant differences in bold.

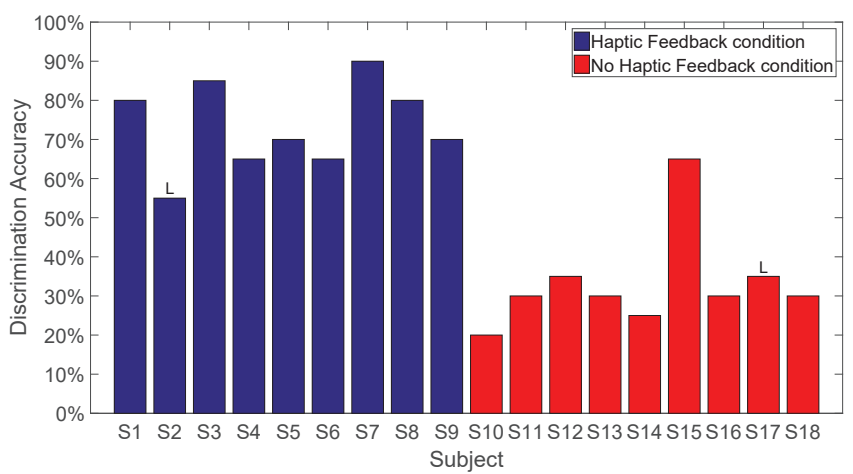

Fig. 6: Discrimination accuracy during the sphere size discrimination test. Left handed subjects marked with "L".

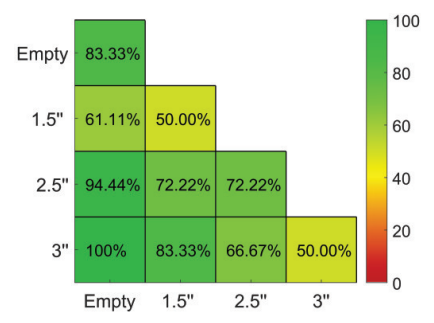

(a) HF condition.

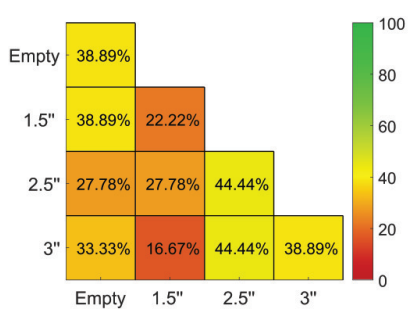

(b) NHF condition
Fig. 7: Pairwise accuracy for the two groups.

during the size discrimination test. Q11 and Q12 show that subjects had good perception of the rotation of the Rocker. Participants who began in the HF condition showed a tendency close to significance to find the Rocker movement easier to detect respect to the NHF subjects $(\mathrm{Q} 11-\mathrm{p}=$ $0.063, \mathrm{Q} 12-\mathrm{p}=0.061$ ), which could be ascribed to the extra training they received. Finally, results for questions Q13 to Q16 show that the experimental conditions were sound, and Q17-18 provide indication that the load on the subjects from the experiment was not excessive.

\section{Conclusions}

In this work we introduce the Rice Haptic Rocker, a skin stretch haptic feedback device that can be used to convey proprioceptive information during opening and closure of a prosthetic hand, moving the skin through an eccentric rocker with frictional contact. We present its integration with the Pisa/IIT SoftHand, which due to its adaptability and simple control is well suited for use with this type of haptic feedback, presenting a setup that, for the first time, integrates skin stretch with a prosthetic device for proprioceptive feedback. We also present an experimental evaluation of the effectiveness of the proposed approach at conveying proprioception, through a size discrimination test performed with 18 able bodied subject, as well as subjective evaluation of the setup obtained by participants through a Likert-scale survey.

Subjects who performed the size discrimination test with the Rice Haptic Rocker were able to successfully discriminate between different sized spheres with an average accuracy of $73.3 \pm 11.2 \%$, well above the $1 / 3$ chance level which was observed in the control group $(33.3 \pm 12.7 \%)$. Results of the survey showed that the setup was considered easy to use and effective by the participants. Because of these results, we believe the proposed solution to be viable in real prosthetic applications: future work will focus on further experimentation to evaluate performance when a distraction task is present, tests with amputees subjects, evaluation of different locations on the arm to convey the haptic feedback (e.g. forearm) and and grasping conditions closer to a real life situation (subjects grasping objects actively instead of them being offered to them).

\section{ACKNOWLEDGMENT}

The authors gratefully acknowledge Matteo Rossi for his valuable advice and Mikaela Juzswik for her unique contribution in the physical realization of some of the equipment used in the experiments. This work was partially supported by the European Community funded project WEARHAP (contract 601165), by the European Commission project (Horizon 2020 research program) SOFTPRO (no. 688857), by the ERC Advanced Grant no. 291166 SoftHands and by the NSF grant IIS-1065497. 


\section{REFERENCES}

[1] S. Millstein, H. Heger, and G. Hunter, "Prosthetic use in adult upper limb amputees: a comparison of the body powered and electrically powered prostheses," Prosthetics and orthotics international, vol. 10, no. 1, pp. 27-34, 1986.

[2] S. L. Carey, et al., "Differences in myoelectric and body-powered upper-limb prostheses: Systematic literature review," J Rehabil Res Dev, vol. 52, no. 3, p. 247, 2015 .

[3] A. Chadwell, et al., "The reality of myoelectric prostheses: Understanding what makes these devices difficult for some users to control,' Frontiers in Neurorobotics, vol. 10, 2016.

[4] C. Pylatiuk, S. Schulz, and L. Döderlein, "Results of an internet survey of myoelectric prosthetic hand users," Prosthetics and orthotics international, vol. 31, no. 4, pp. 362-370, 2007.

[5] E. Biddiss, D. Beaton, and T. Chau, "Consumer design priorities for upper limb prosthetics," Disability and Rehabilitation: Assistive Technology, vol. 2, no. 6, pp. 346-357, 2007.

[6] S. Lewis, et al., "User demands for sensory feedback in upper extremity prostheses," in Medical Measurements and Applications Proceedings (MeMeA), 2012 IEEE International Symposium on. IEEE, 2012, pp. 1-4.

[7] P. D. Marasco, et al., "Robotic touch shifts perception of embodiment to a prosthesis in targeted reinnervation amputees," Brain, vol. 134, no. 3, pp. 747-758, 2011.

[8] E. A. Biddiss and T. T. Chau, "Upper limb prosthesis use and abandonment: a survey of the last 25 years," Prosthetics and orthotics international, vol. 31, no. 3, pp. 236-257, 2007.

[9] A. Ajoudani, et al., "Exploring teleimpedance and tactile feedback for intuitive control of the pisa/iit softhand," Haptics, IEEE Transactions on, vol. 7, no. 2, pp. 203-215, 2014.

[10] A. Ninu, et al., "Closed-loop control of grasping with a myoelectric hand prosthesis: Which are the relevant feedback variables for force control?" Neural Systems and Rehabilitation Engineering, IEEE Transactions on, vol. 22, no. 5, pp. 1041-1052, 2014.

[11] H. J. Witteveen, et al., "Vibro-and electrotactile user feedback on hand opening for myoelectric forearm prostheses," Biomedical Engineering, IEEE Transactions on, vol. 59, no. 8, pp. 2219-2226, 2012.

[12] E. Treadway, et al., "The role of auxiliary and referred haptic feedback in myoelectric control," in World Haptics Conference (WHC), 2015 IEEE. IEEE, 2015, pp. 13-18.

[13] K. Bark, et al., "Rotational skin stretch feedback: A wearable haptic display for motion," Haptics, IEEE Transactions on, vol. 3, no. 3, pp. 166-176, 2010.

[14] K. Kim and J. Colgate, "Haptic feedback enhances grip force control of semg-controlled prosthetic hands in targeted reinnervation amputees," Neural Systems and Rehabilitation Engineering, IEEE Transactions on, vol. 20, no. 6, pp. 798-805, 2012.

[15] D. J. Atkins, D. C. Heard, and W. H. Donovan, "Epidemiologic overview of individuals with upper-limb loss and their reported research priorities." JPO: Journal of Prosthetics and Orthotics, vol. 8, no. 1, pp. 2-11, 1996.

[16] A. Blank, A. M. Okamura, and K. J. Kuchenbecker, "Identifying the role of proprioception in upper-limb prosthesis control: Studies on targeted motion," ACM Transactions on Applied Perception (TAP), vol. 7, no. 3, p. 15, 2010.

[17] S. B. Godfrey, et al., "The softhand pro: Translation from robotic hand to prosthetic prototype," in Converging Clinical and Engineering Research on Neurorehabilitation II. Springer International Publishing, 2017, pp. 469-473.

[18] M. Rossi, et al., "Hap-pro: a wearable haptic device for proprioceptive feedback," submitted to Transactions on Biomedical Engineering, in press, preprint available at: https://goo.gl/lQeEUd.

[19] T. Pistohl, et al., "Artificial proprioceptive feedback for myoelectric control," Neural Systems and Rehabilitation Engineering, IEEE Transactions on, vol. 23, no. 3, pp. 498-507, 2015.

[20] G. Dhillon, et al., "Effects of short-term training on sensory and motor function in severed nerves of long-term human amputees," Journal of neurophysiology, vol. 93, no. 5, pp. 2625-2633, 2005.

[21] J. S. Schofield, et al., "Applications of sensory feedback in motorized upper extremity prosthesis: a review," Expert review of medical devices, vol. 11, no. 5, pp. 499-511, 2014.

[22] S. Micera, J. Carpaneto, and S. Raspopovic, "Control of hand prostheses using peripheral information," Biomedical Engineering, IEEE Reviews in, vol. 3, pp. 48-68, 2010.
[23] R. Christiansen, et al., "Vibrotactile feedback of pose error enhances myoelectric control of a prosthetic hand," in World Haptics Conference (WHC), 2013. IEEE, 2013, pp. 531-536.

[24] C. J. Hasson and J. Manczurowsky, "Effects of kinematic vibrotactile feedback on learning to control a virtual prosthetic arm," Journal of neuroengineering and rehabilitation, vol. 12, no. 1, p. 1, 2015.

[25] K. A. Kaczmarek, et al., "Electrotactile and vibrotactile displays for sensory substitution systems," Biomedical Engineering, IEEE Transactions on, vol. 38, no. 1, pp. 1-16, 1991.

[26] A. Akhtar, et al., "Passive mechanical skin stretch for multiple degree-of-freedom proprioception in a hand prosthesis," in Haptics. Neuroscience, Devices, Modeling, and Applications. Springer, 2014 pp. $120-128$.

[27] J. Wheeler, et al., "Investigation of rotational skin stretch for proprioceptive feedback with application to myoelectric systems," Neural Systems and Rehabilitation Engineering, IEEE Transactions on, vol. 18, no. 1, pp. 58-66, 2010 .

[28] F. Chinello, et al., "Design of a wearable skin stretch cutaneous device for the upper limb," in 2016 IEEE Haptics Symposium (HAPTICS). IEEE, 2016, pp. 14-20.

[29] X. Liang, et al., "[d86] skin-stretch proprioceptive feedback for a robotic gripper," in 2014 IEEE Haptics Symposium (HAPTICS), Feb 2014, pp. 1-1.

[30] E. Murray, et al., "[demo] 2-dof skin-stretch haptic feedback device." in IEEE World Haptics Conference. IEEE, 2015.

[31] A. Blank, et al., "[work in progress] skin stretch feedback of gripper aperture for prosthetic hands in a grasp and lift task." in IEEE World Haptics Conference. IEEE, 2015.

[32] S. B. Godfrey, et al., "A synergy-driven approach to a myoelectric hand," in 13TH International Conference on Rehabilitation Robotics, June 24-26, 2013, Seattle, WA., 2013, pp. 1 - 6. [Online]. Available: 10.1109/ICORR.2013.6650377

[33] S. Fani, et al., "Assessment of myoelectric controller performance and kinematic behavior of a novel soft synergy-inspired robotic hand for prosthetic applications," Frontiers in Neurorobotics, vol. 10, p. 11, 2016. [Online]. Available: http://journal.frontiersin.org/article/ 10.3389/fnbot.2016.00011

[34] S. Casini, et al., "Design and realization of the cuff-clenching upperlimb force feedback wearable device for distributed mechano-tactile stimulation of normal and tangential skin forces," in Intelligent Robots and Systems (IROS), 2015 IEEE/RSJ International Conference on. IEEE, 2015, pp. 1186-1193.

[35] S. B. Godfrey, et al., "Influence of force feedback on grasp force modulation in prosthetic applications: A preliminary study," in Engineering in Medicine and Biology Society (EMBC), 2016 IEEE 38th Annual International Conference of the. IEEE, 2016, pp. 5439-5442.

[36] A. Ajoudani, et al., "Exploring teleimpedance and tactile feedback for intuitive control of the pisa/iit softhand," IEEE transactions on haptics, vol. 7, no. 2, pp. 203-215, 2014.

[37] M. G. Catalano, et al., "Adaptive synergies for the design and control of the pisa/iit softhand," The International Journal of Robotics Research, vol. 33, no. 5, pp. 768-782, 2014.

[38] M. Santello, M. Flanders, and J. F. Soechting, "Postural hand synergies for tool use," The Journal of Neuroscience, vol. 18, no. 23, pp. 10105 $10115,1998$.

[39] P. H. Thakur, A. J. Bastian, and S. S. Hsiao, "Multidigit movement synergies of the human hand in an unconstrained haptic exploration task," The Journal of neuroscience, vol. 28, no. 6, pp. 1271-1281, 2008.

[40] A. Bicchi, M. Gabiccini, and M. Santello, "Modelling natural and artificial hands with synergies," Philosophical Transactions of the Royal Society of London B: Biological Sciences, vol. 366, no. 1581, pp. 3153-3161, 2011. 